\title{
Isolated oculocutaneous sarcoidosis in a teenage male: a rare case report
}

\author{
H.K. Aggarwal, D. Jain, A. Mittal, G. Dabas \\ Department of Medicine, Sharma University of Health Sciences, Rohtak, India
}

\section{SUMMARY}

Sarcoidosis is an inflammatory disease with multisystem involvement characterized by the presence of noncaseating granulomas. It can affect virtually every organ of the body, with lung involvement being most common occurring in $>90 \%$ of patients. Other organs affected are skin, eye and liver. Skin involvement is common, affecting $25-35 \%$. Here we present a rare case of a 15 year-old male with isolated oculocutaneous sarcoidosis without systemic involvement.

Key words: Sarcoidosis; Non-caseating granulomas; Oculocutaneous granulomatosis.

Reumatismo, 2016; 68 (2): 100-103

\section{INTRODUCTION}

arcoidosis is a multisystem granulomatous disorder of unknown etiology characterized by presence of non-caseating granulomas. The main organ affected is the lung. $30 \%$ of patients have extra pulmonary sarcoid with involvement of lymph node, liver and spleen. Sarcoidosis has variable prevalence worldwide ranging between 30 and 60 per 100,000 people. It mainly affects young adults and is uncommon to be diagnosed in patients younger than 18 years.

\section{CASE REPORT}

A 15 year-old male patient, non-smoker, non-alcoholic, vegetarian, presented with chief complaints of skin lesions on the dorsal aspect of both hands since 2 months that were reddish in color, not associated with pain, ulcerations or photosensitivity. There was no history of fever, cough with or without expectoration, night sweats, loss of appetite or weight, shortness of breath, weakness, joint pains, hair fall, oral ulcer, dry eyes, photophobia, watering eye or decreased vision.
Skin examination revealed erythematous patches on the dorsum of the hands, which were multiple, well defined, soft, flat, with smooth surface, and variable size varying from $1.5 \times 1.5$ to $4 \times 4 \mathrm{~cm}$ (Figure $1 \mathrm{~A}$ ). On laboratory investigations complete hemogram, renal and hepatic functions were within normal limits.

Erythrocyte sedimentation rate was 20 $\mathrm{mm} / \mathrm{hr}$ and of 1st hour and Mantoux test was negative. Further biochemical investigations revealed corrected serum calcium of $9.1 \mathrm{mg} / \mathrm{dL}$, serum phosphate $3.5 \mathrm{mg} /$ $\mathrm{dL}$ and serum alkaline phosphatase 83 U/L. Serum acetylcholine esterase (ACE) level was elevated with an absolute value of $140 \mathrm{U} / \mathrm{L}(8-65 \mathrm{U} / \mathrm{L})$. Anti-nuclear antibodies and other autoimmune disease markers like antineutrophil cytoplasmic antibodies (c ANCA, p ANCA), antibodies to double-stranded DNA (anti dsDNA), anti SS-A(Ro) antibodies and anti SSB(La) antibody were negative. Chest $\mathrm{X}$ ray, ultrasound of the abdomen, pulmonary function test and echocardiography were normal.

Slit lamp examination of the eye showed granulomatous iridocyclitis, mutton fat precipitates, iris nodules with posterior synechiae (Figure 1B). Fundus examination was normal. High-resolution computed tomography (HRCT) and contrast enhanced computed tomography of the chest and magnetic resonance of the brain were also normal (Figure 2A, B). On bronchoalveolar lavage (BAL) CD4:CD8 ratio was 


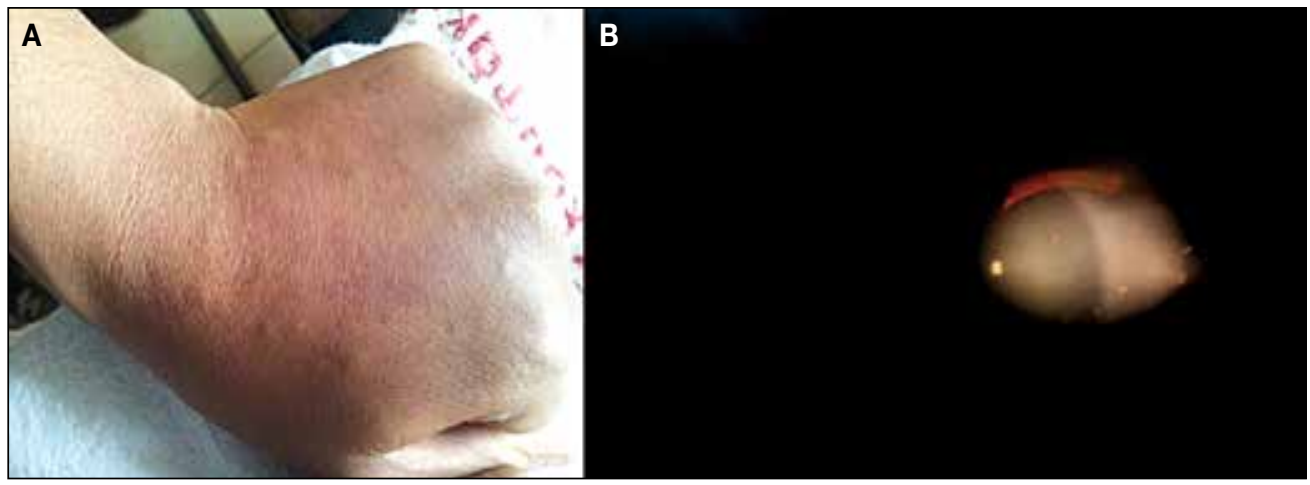

Figure 1 - A) Multiple, well defined, soft, flat, smooth surface, variable size (from $1.5 \times 1.5$ to $4 \times 4$ $\mathrm{cm})$, erythematous lesions and patches on dorsum of hand; B) slit lamp examination of eye.

$<2$ ruling out any pulmonary involvement. Skin biopsy revealed mildly atrophic epidermis with upper dermis showing mild perivascular lympho-mononuclear infiltrates and deep dermis revealed naked non-caseating epithelioid cell granulomas, which consist of aggregates of epithelioid histiocytes, giant cells and mature macrophages without significant lymphocytic infiltrate and absence of Langerhans giant cells. Staining for acid-fast bacillus was negative (Figure 3).

The patient was treated with topical steroids for skin and ocular lesions. After one month of therapy there was partial improvement in skin lesions and the iridocyclitis was in healing phase. The patient was continued on therapy and kept on regular follow up to monitor for other systemic involvement.

\section{DISCUSSION}

Sarcoidosis is a multisystem disorder of unknown etiology characterized by presence of non-caseating granulomas. It occurs worldwide with highest prevalence in the Scandinavian population. The highest annual incidence of sarcoidosis has been observed in northern European countries ( 5 to 40 cases per 100,000 people) (1). It commonly affects young adults with a second peak in incidence occurring around age 60 . It mainly involves lung and up to $30 \%$ patients present with extra pulmonary sarcoidosis $(2,3)$. Lesions of sarcoidosis

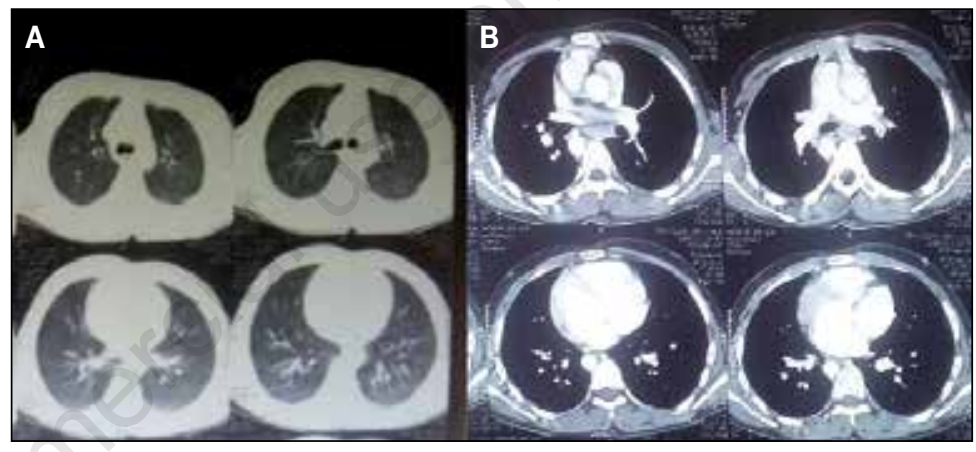

Figure 2 - High-resolution computed tomography and contrast enhanced computed tomography of the chest.

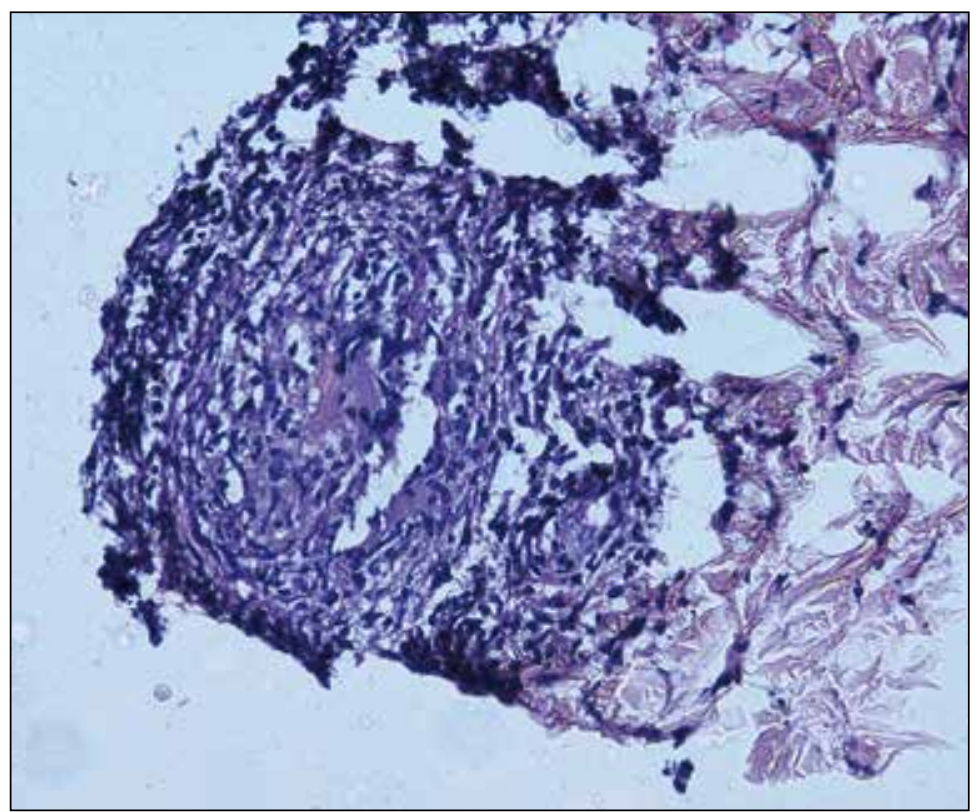

Figure 3 - Acid-fast bacillus test of a skin biopsy. 
manifest in the lung in form of bilateral hilar lymphadenopathy, peribronchial thickening and reticulo-nodular changes. Patient can be asymptomatic, detected incidentally on routine $\mathrm{X}$ ray or they may present with chronic dry cough. The most common skin lesions are erythema nodosum, plaques, maculopapular eruptions, subcutaneous nodules, and lupus pernio (4). Skin lesions are often overlooked, as these are chronic and not painful.

The most common involvement of the eye is anterior uveitis, but retinitis and pars planitis can also occur. Chronic anterior uveitis is usually associated with iris nodules. Although Busacca nodules are true granulomatous lesions found on the iris, a Koeppe nodule is a granulomatous lesion found only on the pupillary margin. It may become the nidus for posterior synechiae (2). These patients can present with photophobia, increased lacrimation and blurred vision or they can be asymptomatic even with active inflammation. Such patients are at risk of losing their vision if early diagnosis is not made. Other organs are less commonly involved in sarcoidosis. Pathologically, sarcoidosis is characterized by formation of non-caseating granulomas in the affected organ, which are formed as a result of activation of cellular immune response to antigen exposure.

The exact etiology behind sarcoidosis is unknown but there are hypotheses suggesting a role for environmental factors initiating an immunological response in a genetically susceptible individual. Some viral and bacterial infections like mycobacteria, Epstein-Barr virus and human herpesvirus- 8 are implicated.

The initial inflammatory response is an influx of T helper cells associated with an accumulation of activated monocytes. Using the HLA-CD4 complex, antigen-presenting cells present an unknown antigen to the helper $\mathrm{T}$ cell, which leads to their activation. T-helper cells produce chemokines and cytokines, which cause amplification of cellular immune response and migration of mononuclear phagocytes and other inflammatory cells into the tissues (5-7).
The diagnosis of sarcoidosis is based mainly on clinical characteristics, radiological findings, histopathology and exclusion of other non-caseating granuloma forming conditions (8).

Certain criteria have been proposed to support the diagnosis of definite or probable ocular sarcoidosis in a patient with known sarcoidosis (9). On the other hand, like in our case, patients may present with ocular findings compatible with sarcoidosis but no symptoms from extra ocular disease. In this situation, several groups have tried to identify features that support ocular sarcoidosis, including roentgenographic findings on HRCT or cellular elements in the BAL. However, the gold standard for definitive diagnosis remains biopsy confirmation. This approach has been supported by one retrospective case-controlled study of Japanese patients (10). Hematological and biochemical tests may show normal erythrocyte sedimentation rate, anemia, lymphocytopenia, mildly increased liver enzymes, hypercalcemia and increased serum angiotensin enzyme levels. Serum chitotriosidase is a sensitive and reproducible biomarker of sarcoidosis as well as a reliable bio indicator of disease severity and is increased in over $90 \%$ of patients with active disease (11).

The diagnosis is further supported by the histological appearance of the non-caseating epitheloid cell granulomas in affected organs or tissues. Schaumann bodies, which contain calcium oxalate crystals, are found in 48 to $88 \%$ of the patients. Asteroid or satellite bodies are interesting occasional findings seen in less than 2 and $9 \%$ of the cases (12).

Cutaneous lesions of sarcoid are often missed because they mimic many common skin conditions like lupus vulgaris, psoriasis, lichen planus etc. Cutaneous sarcoidosis has been reported rarely from India with a much lesser incidence in childrens (13). Similarly isolated ocular involvement is very uncommon in sarcoidosis. Ocular lesions, if missed, can cause severe visual impairment or even blindness in the absence of adequate treatment. Uveitis precedes the non-ocular signs of sarcoidosis 
in approximately $30 \%$. So a high degree of suspicion is necessary for diagnosing this condition because early diagnosis and intervention can prevent significant morbidity and mortality. These patients are highly likely to develop other organ involvement so they must be kept on regular follow up for timely detection and intervention as required.

The optimal treatment of sarcoidosis depends on the location of the disease and severity of involvement. The mainstay of treatment is corticosteroids. For refractory cases, methotrexate has been found to be effective. Ocular and cutaneous lesions are usually treated with topical steroids and have good prognosis with treatment. Systemic steroids are indicated in optic neuritis and topical treatment resistant posterior uveitis.

Our patient presented with characteristic findings on eye examination along with confirmation on skin biopsy and biochemical evidence of increased ACE levels after exclusion of tuberculosis. The present case demonstrated an isolated oculocutaneous sarcoid without pulmonary involvement in a teenage male, which has not been reported the literature. These cases, later on, can progress to involve respiratory system thus leading to increased morbidity, hence highlighting the issue of repeated follow-up to prevent pulmonary and ophthalmological complications.

\section{CONCLUSIONS}

Sarcoidosis is a great emulator of many dermatological diseases since it has a variable presentation. Great awareness about this presentation of sarcoidosis helps in early diagnosis as most of these patients progress to have systemic involvement giving us an opportunity to instate early therapy.

Conflict of interest: the authors declare no potential conflict of interest.

\section{REFERENCES}

1. Pietinalho A, Hiraga Y, Hosoda Y, et al. The frequency of sarcoidosis in Finland and Hokkaido, Japan: a comparative epidemiological study. Sarcoidosis. 1995; 12: 61-7.

2. Baughman RP, Teirstein AS, Judson MA, et al. Clinical characteristics of patients in a case control study of sarcoidosis. Am J Respir Crit Care Med. 2001; 164: 1885.

3. Rizzato G, Tinelli C. Unusual presentation of sarcoidosis. Respiration. 2005; 72: 3.

4. Mana J, Marcoval J. Skin manifestations of sarcoidosis. Presse Med. 2012; 41: e355-74.

5. Fernandez Fabrellas E. Epidemiology of sarcoidosis. Arch Bronconeumol. 2007; 43: 92-100.

6. English JC III, Patel PJ, Greer KE. Sarcoidosis. J Am Acad Dermatol. 2001; 44: 725-43.

7. Moller DR, Chen ES. What causes sarcoidosis. Curr Opin Pulm Med. 2002; 8: 429-34.

8. Müller-Quernheim. Serum markers for the staging of disease activity of sarcoidosis and other interstitial lung diseases of unknown etiology. J Sarcoidosis Vasc Diffuse Lung Dis. 1998; 15: 22-37.

9. Herbort CP, Rao NA. International criteria for the diagnosis of ocular sarcoidosis: results of the first International Workshop on Ocular Sarcoidosis (IWOS). Ocul Immunol Inflamm. 2009; 17: 160-9.

10. Kawaguchi T, Hanada A, Horie S, et al. Evaluation of characteristic ocular signs and systemic investigations in ocular sarcoidosis patients. Jpn J Ophthalmol. 2007; 51: 121-6.

11. Harlander M, Salobir B, Zupancic M, et al. Serial chitotriosidase measurements in sarcoidosis-two to five year follow-up study. Respir Med. 2014; 108: 775-82.

12. Gal AA, Koss MN. The pathology of sarcoidosis. Curr Opin Pulm Med. 2002; 8: 445-51.

13. Rajam RV, Vishwanathan GS, Rangaiah PN, et al. Sarcoidosis- a short review with a case report. Indian Dermatol Venereol Leprol. 1957; 23: 97-135. 